Broman-Fulks, J. J., Ruggiero, K. J., Green, B.A., Kilpatrick, D. G., Danielson, C.K., Resnick, H. S., \& Saunders, B. E. (2006). Taxometric investigation of PTSD: Data from two nationally representative samples. Behavior Therapy, 37(4): 364-380. (Dec 2006) Published by Elsevier (ISSN: 1878-1888). doi:10.1016/j.beth.2006.02.006

\title{
Taxometric Investigation of PTSD: Data From Two Nationally Representative Samples
}

Joshua J. Broman-Fulks, Kenneth J. Ruggiero, Bradley A. Green, Dean G. Kilpatrick, Carla Kmett Danielson, Heidi S. Resnick, Benjamin E. Saunders

\begin{abstract}
Current psychiatric nosology depicts posttraumatic stress disorder (PTSD) as a discrete diagnostic category. However, only one study has examined the latent structure of PTSD, and this study suggested that PTSD may be more accurately conceptualized as an extreme reaction to traumatic life events rather than a discrete clinical syndrome. To build on the existing literature base, the present research examined the latent structure of posttraumatic stress reactions by applying three taxometric procedures (MAXEIG, MAMBAC, and L-Mode) to data collected from large nationally representative samples of women ( $n s=2684$ and 3033 ) and adolescents $(n=3775)$. Results consistently provided evidence for a dimensional PTSD solution across samples and statistical procedures. These findings have important implications for the theory, assessment, and investigation of posttraumatic stress reactions.
\end{abstract}


The conceptualization, measurement, and approach to studying emotional and behavioral reactions to potentially traumatic events is fundamentally informed by the nature of the relation between posttraumatic stress disorder (PTSD) and normal stress reactions. Yet, considerable debate exists over whether PTSD represents a discrete clinical syndrome or the upper end of a stress-response continuum (e.g., Ruscio, Ruscio, \& Keane, 2002). An examination of the current psychiatric nosology, as represented by the fourth edition of the Diagnostic and Statistical Manual of Mental Disorders (American Psychiatric Association, 1994), reveals a general assumption that pathological posttraumatic stress reactions should be distinguished from "normal" responses to extreme stressors. Furthermore, many organizations (e.g., insurance companies, Departments of Veteran Affairs) determine who will receive access to mental health services and who is eligible for disability compensation based on the presence or absence of the psychiatric diagnosis. However, recent research has suggested that the boundary between normal and pathological responses to extreme stress may not be quite so clear cut.

Epidemiological studies have indicated that approximately $55 \%$ to $70 \%$ of people experience some form of violent or traumatic victimization in their lifetime ( [Kessler et al., 1995] , [Norris, 1992] and [Resnick et al., 1993] ). In response to traumatic events, a significant percentage of individuals experience at least some symptoms of PTSD (Rothbaum, Foa, Riggs, Murdock, \& Walsh, 1992), and in many cases some symptoms are present even 1 year later (Veronen \& Kilpatrick, 1983). Although only $4 \%$ to $12 \%$ of victims ever meet the diagnostic threshold for PTSD ( [Kessler et al., 1995] , [Kilpatrick et al., 2003] and [Resnick et al., 1993] ), research has revealed that subsyndromal or partial presentations of PTSD are often associated with clinically significant impairment in psychosocial and occupational functioning (Marshall et al., 2001). Furthermore, patterns of help-seeking behavior among individuals with partial PTSD are similar to those of individuals who meet full diagnostic criteria (e.g., Stein, Walker, Hazen, \& Forde, 1997). Thus, questions have been raised concerning the clinical utility of the PTSD diagnosis and whether PTSD reactions are qualitatively or quantitatively different from subthreshold posttraumatic stress reactions (i.e., if the latent structure of PTSD is taxonic or dimensional).

A taxon is a nonarbitrary latent category whose members are qualitatively distinct from nonmembers (Haslam, 2003). Nature and society are known to contain numerous taxa, including chemical elements, biological species, and occupational categories. In contrast, nontaxonic phenomena, such as intelligence, age, temperature, or height, are characterized by continuous or dimensional latent structure. Research examining the latent structure of PTSD (i.e., the boundaries differentiating PTSD from normal stress reactions) has the potential to advance our understanding of posttraumatic stress reactions in several important ways. First, research of this nature advances knowledge relating to the development and selection of assessment instruments that correspond well with the latent structure of PTSD. The psychometric strategy behind the construction of assessment instruments is very different when one is attempting to assign individuals to a category versus when one is trying to pinpoint an individual's location on a dimension. In the case of a dimensional variable, the goal of assessment measures is to disperse scores broadly and to discriminate effectively in all areas of the dimension. In contrast, test construction for a taxonic or categorical variable would aim to sort individuals at an optimal cut, in an attempt to maximize valid classifications (see Grove, 
1991, for an extended discussion on this point). Second, research aimed at identifying the most effective diagnostic and treatment methods is enhanced when the underlying structure of the targeted pathology is known. Third, knowing the latent structure of a variable can help to inform etiological research. Specifically, taxonic latent structure is suggestive of a discrete etiological source, such as a particular gene, brain disorder, environmental stressor, or specific interaction of such variables. In contrast, dimensional structure implies an additive or graded etiology (i.e., it is multiply-determined). Thus, with regard to posttraumatic stress reactions, dimensional latent structure would suggest that various trauma-related and person-specific factors (e.g., perpetrator violence, victim's interpretation, traumatic event history) may be important to consider in determining PTSD etiology.

Taxometrics is a branch of applied mathematics designed specifically to discern the latent structure (i.e., categorical versus continuous distribution) of phenomena by evaluating whether the numerical relations among various indicators of a conjectured taxon fit a pattern consistent with a naturally occurring class or a continuous dimension (Meehl \& Golden, 1982). Taxometric procedures have a long history in the literature (e.g., Meehl, 1973) and have recently gained popularity as their utility has become increasingly recognized. Researchers have used these procedures to examine the latent structure of numerous forms of psychopathology, including schizotypy (e.g., Korfine \& Lenzenweger, 1995), bulimia nervosa (Gleaves, Lowe, Snow, Green, \& Murphy-Eberez, 2000), and depression (e.g., [Ruscio and Ruscio, 2000] and [Ruscio and Ruscio, 2002] ). With regard to PTSD, only one taxometric investigation is available in the published literature. Ruscio et al. (2002) examined the latent structure of PTSD with a sample of 1230 male combat veterans participating in the context of a psychological treatment evaluation. Results from this analysis provided support for a dimensional solution, which suggests that PTSD may be better characterized as reflecting the upper end of a posttraumatic stress continuum rather than a discrete psychiatric disorder.

Findings reported by Ruscio et al. (2002) represent an important first step in understanding the latent structure of responses to potentially traumatic events in a veteran combat population. However, taxometricians generally agree that conclusions regarding taxonicity or dimensionality should not be based on any single analysis (Waller \& Meehl, 1998). Rather, replication of results across various samples, assessment measures, and taxometric procedures is required before definitive conclusions regarding the latent structure of a variable can be made. The purpose of this report is to replicate and extend the Ruscio et al. analyses by conducting three studies with data from two large nationally representative samples: (a) the National Women's Study (NWS) Waves I and II (nNWS-I = 2684; nNWS-II = 3033), and (b) the National Survey of Adolescents (NSA; $n=3775$ ). Data from these samples build on findings of Ruscio et al. in several meaningful ways. First, the Ruscio et al. sample consisted exclusively of combat veterans. In contrast, the NWS-I sample was comprised of participants with a wide range of victimization histories (e.g., rape, physical assault, natural disaster, witnessed serious injury), and the NWS-II and NSA samples included both victimized and nonvictimized participants. Second, NWS and NSA data are from nationally representative samples that yielded distributions of PTSD reactions that may generalize well to the U.S. population of women and adolescents, whereas combat samples typically have a substantially higher prevalence of PTSD diagnoses (68\% in the Ruscio et al. sample). Third, the present study includes both female 
(NWS-I, NWS-II, NSA) and male (NSA) respondents and covers a broad age range (MNWS = 41.0 years; MNSA = 14.5 years). In contrast, the Ruscio et al. sample was comprised solely of adult men with a high median age (48 years; mean not reported). Fourth, the structured diagnostic interview for PTSD used in the present study differs from that used by Ruscio and colleagues. In sum, we attempted to extend the findings of Ruscio and colleagues' study using multiple large, representative samples comprised of adults, children, and both genders, as well as a different measure of PTSD.

Based on the Ruscio et al. (2002) findings, we hypothesized that multiple taxometric procedures would yield a continuous solution across two large civilian samples. It is also noteworthy that Study 3 represents one of the first taxometric investigations of a psychiatric condition in an adolescent population.

\section{STUDY 1}

\section{METHOD}

\section{Participants}

Participants were selected for the NWS-I using a multi-stage, stratified, random-digit dialing procedure within the four regions of the country (see Resnick et al., 1993). The full sample ( $\mathrm{N}=$ 4008) included a national household probability sample of 2008 women and an oversample of 2000 women aged 18 to 34 years. To correct for oversampling, data were weighted on the basis of age and race to be consistent with 1989 Census distributions of these characteristics in the U.S. population of women. Mean age of the weighted sample was 44.9 years $(S D=18.4)$. Racial/ethnic distribution was as follows: 80.7\% Caucasian, 11.0\% African American, 5.4\% Hispanic, 1.7\% Native American, and 1.1\% Asian. Lifetime prevalence of victimization experiences included: $12.6 \%$ for completed rape, $14.3 \%$ for other types of sexual assault, $10.3 \%$ for physical assault, $13.4 \%$ for homicide of a family member, and $33.3 \%$ for non-crimerelated disasters (e.g., natural disasters, motor vehicle accidents). Further details on demographics and victimization histories are reported by Resnick et al. (1993).

\section{Measure}

PTSD reactions were assessed using the PTSD module of the NWS survey (Kilpatrick, Resnick, Saunders, \& Best, 1989), a structured diagnostic interview that assessed each DSM-IV criterion with a yes/no response. Research on the NWS-PTSD module has provided support for concurrent validity and several forms of reliability (e.g., temporal stability, internal consistency, diagnostic reliability), as detailed elsewhere (e.g., [Kilpatrick et al., 2003] , [Resnick et al., 1993] and [Ruggiero et al., 2003] ). Notably, the NWS-PTSD module was validated in a field trial against the PTSD module of the Structured Clinical Interview for the DSM (Spitzer, Williams, Gibbon, \& First, 1992) administered by mental health professionals. In the field trial, the 
interrater kappa coefficient was 0.85 for the diagnosis of PTSD, and comparisons between the NWS-PTSD module and SCID yielded a kappa coefficient of 0.77 (Kilpatrick et al., 1998).

\section{PROCEDURE}

\section{Data collection}

The structured telephone interview (of which the PTSD Module was a small component) took approximately 35 minutes to complete. The interview was administered by trained female interviewers employed by Schulman, Ronca, and Bucuvalas, Inc. (SRBI), a survey research firm with significant experience managing survey studies. A computer-assisted telephone interview system aided this process by prompting interviewers with each question consecutively on a computer screen, and supervisors conducted random checks of data entry accuracy and interviewers' adherence to assessment procedures. The NWS-PTSD module was administered to all women who endorsed one or more forms of violent or traumatic victimization (e.g., rape, physical assault, witnessed injury or death, motor vehicle accident). This resulted in a study subsample of 2684 women (67\% of the full sample) who endorsed at least one form of victimization and provided responses to all PTSD symptom questions. Because victimized women were, on average, approximately 5 years younger than nonvictimized women, this subsample was slightly younger than the full sample. However, the subsample did not differ from the full sample with regard to racial/ethnic distribution.

\section{Indicator selection}

Current psychiatric nosology (i.e., DSM-IV) suggests that individuals can be divided into pathological and nonpathological posttraumatic stress categories on the basis of three symptom clusters-reexperiencing, avoidance/numbing, and hypervigilance. Thus, three composite indicators were created for the present study by summing the items on the NWS-PTSD that corresponded with items on each of the three DSM-IV symptom clusters. Table 1 displays the NWS-PTSD items that were summed to form the three PTSD indicators. In addition to ensuring an adequate representation of the full PTSD construct, the summation of these items increased the number of potential response points, thus improving the reliability of the resulting curves and producing indicators that were similar to those used by Ruscio et al. (2002). 
Table 1

Items selected from the NWS-PTSD and summed to create composite indicators

\begin{tabular}{|c|c|}
\hline & Has there ever been a period of one month or more during which ... \\
\hline $\begin{array}{l}\text { Indicator } 1 \\
\text { Reexperiencing }\end{array}$ & $\begin{array}{l}\text { (1) disturbing memories kept coming into your mind whether you wanted to think of them or not? } \\
\text { (2) you had repeated bad dreams or nightmares? } \\
\text { (3) Have you ever had a "flashback" - that is, have you ever had an experience in which you imagined that } \\
\text { something that happened in the past was happening all over again? } \\
\text { (4) you felt a lot worse when you were in a situation that reminded you of something that had happened in the past? } \\
\text { (5) you found yourself reacting physically to things that reminded you of something that had happened to you in the } \\
\text { past - like breaking out in a sweat, breathing heavily or irregularly, or heart pounding or racing? }\end{array}$ \\
\hline $\begin{array}{l}\text { Indicator } 2 \\
\text { Avoidance/Numbing }\end{array}$ & $\begin{array}{l}\text { (1) you deliberately tried very hard not to think about something that had happened to you? } \\
\text { (2) you went out of your way to avoid certain places or activities which might remind you of something that had } \\
\text { happened to you in the past? } \\
\text { (3) Have you ever felt that there were parts of any such experiences that you couldn't remember? } \\
\text { (4) you lost interest in activities which usually meant a lot to you? } \\
\text { (5) you felt cut off from other people or found it difficult to feel close to other people? } \\
\text { (6) it seemed you could not feel things anymore or that you had much less emotion than you used to? } \\
\text { (7) the way you think about or plan for the future was changed by something that happened to you in the past? }\end{array}$ \\
\hline $\begin{array}{l}\text { Indicator } 3 \\
\text { Hyperarousal }\end{array}$ & $\begin{array}{l}\text { (1) you had difficulty falling asleep or staying asleep? } \\
\text { (2) little things bothered you a lot or could make you very angry? } \\
\text { (3) you had trouble concentrating or keeping your mind on what you were doing, even when you tried to } \\
\text { concentrate? } \\
\text { (4) you felt you had to stay on guard much of the time? } \\
\text { (5) unexpected noises startled you more than usual? }\end{array}$ \\
\hline
\end{tabular}

Note. Items were selected based on DSM-IV diagnostic criteria for posttraumatic stress disorder.

Meehl and Yonce (1994) stated that "each indicator should be selected to . . . be uncorrelated within either the taxon or the complement group with the other variable(s) being used, i.e., to have no nuisance covariance" (p. 1061). Thus, the mean within-groups correlations among the indicators were examined for each study. Nuisance covariances proved to be extremely low $(0.02,0.03$, and 0.00 for Studies 1,2 , and 3 , respectively). In addition, it is generally recommended that indicators with high degrees of validity (i.e., 1.25 SD or greater separation between conjectured taxon and nontaxon members) be used in taxometric analyses (Meehl, 1995). Indicators employed in the present study were highly valid, with mean separations of 3.31, 3.45, and 3.11 SD for Studies 1, 2, and 3, respectively. Thus, the indicators utilized in the present research are appropriate for taxometric analysis.

\section{Statistical procedures}

We employed three mathematically independent taxometric procedures to evaluate the latent structure of PTSD: MAMBAC (mean above minus below a cut; see Meehl \& Yonce, 1994), MAXEIG (maximum eigenvalue; [Meehl and Yonce, 1996] and [Waller and Meehl, 1998] ), and L-mode (Latent-mode factor analysis; Waller \& Meehl, 1998). Following is a brief explanation of the mathematical rationale behind each procedure. All MAXEIG, MAMBAC, and L-Mode analyses were run using S+ statistical software using algorithms obtained from Ruscio (2003). 


\section{MAXEIG}

MAXEIG (Waller \& Meehl, 1998) is a multivariate extension of the frequently used MAXCOV procedure (Meehl \& Yonce, 1996). MAXEIG calculates and plots eigenvalues from all remaining indicators across successive intervals of an input indicator. Each indicator serves as the input indicator on one analysis resulting in one MAXEIG plot per indicator. If a taxon is present, data typically yield plots with peaked curves. In contrast, dimensional data tend to produce relatively flat plots. Recent research has demonstrated that the traditional MAXCOV procedure is particularly susceptible to imperfections in the distributional characteristics of the data (Ruscio \& Ruscio, 2002). Specifically, when indicators with skewed distributions are subjected to MAXCOV, plots produced by dimensional data can be easily misinterpreted as plots generated by a low base rate taxon. In contast, MAXEIG generates a far greater number of response points and is more robust to imperfections in the data (Ruscio, Ruscio, \& Keane, 2004). Thus, the current research utilized the MAXEIG procedure to ensure accurate plot interpretation, particularly since relatively low base rates were expected among the nationally representative samples. The current research also included the MAXEIG Inchworm Consistency Test (ICT; Waller \& Meehl, 1998), using each indicator as an input variable with 50, 75, and 100 windows and an overlap of 0.90 . The ICT increases the interpretability of MAXEIG plots and is particularly beneficial for elucidating the presence of extreme high or low base rate taxa (see Waller \& Meehl, 1998).

\section{MAMBAC}

The MAMBAC (Meehl \& Yonce, 1994) procedure is based on the assumption that if two discrete groups exist (i.e., taxon and nontaxon), mean differences between groups will occur on valid indicators of group membership. When mean differences are plotted as a function of one variable, taxonic constructs tend to produce peaked plots at the point that best differentiates the two groups, whereas dimensional constructs produce dish-shaped plots. It should be noted that the present study used a slightly modified version of the MAMBAC procedure. Instead of using all possible pairs of indicators as the input and output variable (as is typical in MAMBAC analyses), the modified MAMBAC procedure uses each indicator as an output variable on one plot, with the factor scores of the first unrotated factor of the remaining indicators serving as the input variable. The modified MAMBAC procedure was used to improve interpretability of the resulting data plots by increasing the potential variability in the input variable, thus creating a more stable curve.

\section{L-Mode}

L-mode (Waller \& Meehl, 1998) combines all candidate indicators and conducts an exploratory factor analysis on the covariances between indicators. Factor score estimates for the first unrotated factor are computed and used to plot a factor-score probability density distribution (Arnau, Thompson, \& Cook, 2001). The distribution of scores for the first unrotated factor is then 
examined. If the construct under investigation is taxonic, a bimodal distribution of factor scores will be evident. In contrast, if the variable is dimensional, factor scores will be unimodally distributed.

\section{Taxometric graph ratings}

To ensure rating accuracy, taxometric plots were rated as indicative of taxonicity, continuity, or ambiguity by two independent examiners: a doctoral-level psychologist and an advanced clinical psychology graduate student. Both examiners were experienced in taxometric plot ratings and had no a priori assumptions regarding the latent structure of posttraumatic stress reactions. Raters were provided with Waller and Meehl's (1998) recommendations for rating MAXEIG and L-Mode plots and Meehl and Yonce's (1994) guidelines for rating MAMBAC plots. Prior research has indicated that taxometric graphs can generally be rated with greater than 95\% accuracy ( [Meehl and Yonce, 1994] and [Meehl and Yonce, 1996] ) and that interrater agreement is typically high (Gleaves et al., 2000). In addition, plots generated from simulated taxonic and dimensional data with similar distributional characteristics were made available to the raters. Raters were encouraged to compare research data plots with simulated taxonic and dimensional plots and to use these comparisons as an additional source of information in making their decision about the shape of the empirical data plots. The two independent raters in the present study were in perfect agreement (100\%) on all of their plot ratings.

Simulations were created using algorithms developed by Ruscio (2003). Simulated dimensional data are created using an iterative procedure that matches the exact distributions of all indicators including their skew, kurtosis, and discrete values. In contrast, simulated taxonic data sets are generated by employing a fallible criterion variable, denoting the putative class membership of each case, to isolate taxon and complement distributions. The same iterative procedure is then employed to create simulated taxon and complement distributions, which are merged to produce the simulated data set for the taxonic case. The grand mean base rate estimate is used to separate cases into putative taxon versus nontaxon membership. (A detailed description of Ruscio's SimDim and SimTax programs can be found at: http://www2.etown.edu/psychology/Documents/TaxProgDocR.PDF.)

\section{Multiple hurdles consistency tests}

Taxometric methods do not rely on traditional significance testing. Rather, a multiple hurdles consistency testing approach is recommended as part of the coherent cut kinetics method to protect against spurious results ( [Meehl, 1995] and [Waller and Meehl, 1998] ). Thus, the present study employed a series of consistency tests to ensure valid results. First, three mathematically independent taxometric procedures-MAXEIG, MAMBAC, and L-mode-were used to analyze the latent structure of PTSD, each of which serves as a consistency test for the other procedures. Second, two nonredundant samples of participants, comprised of individuals both with and without exposure to traumatic events (see Studies 2 and 3), were used, which 
allowed for an evaluation of the full spectrum of PTSD. The final consistency test involved the examination of base rate estimates generated by the various procedures. Each taxometric procedure generates one or more base rate estimates that also can be examined for consistency both within and across procedures. Inconsistent base rate estimates are considered evidence for the absence of a latent taxon (Waller \& Meehl, 1998).

\section{RESULTS}

The purpose of Study 1 was to examine the latent structure of PTSD in a national sample of women with a history of one or more potentially traumatic experiences. MAXEIG analyses were run first on the NWS-1 data. In addition, the ICT was included in MAXEIG analyses to guard against the nondetection of an extremely low or high base rate taxon. The MAXEIG procedure with ICT generated nine plots, of which seven were rated as dimensional and two were rated as ambiguous. The ICT provided additional evidence for a dimensional solution, with MAXEIG plots increasing in variability around a mean eigenvalue as the number of windows increased. When the NWS-I plots were compared to those produced by simulated taxonic and dimensional data with comparable degrees of skew, the plots produced by the PTSD data resembled those generated by the simulated dimensional data. Refer to Fig. 1 for MAXEIG, MAMBAC, and Lmode plots based on the NWS-I data. Fig. 1 also provides comparison MAXEIG, MAMBAC, and L-mode plots generated by simulated dimensional and taxonic data with distributional properties similar to the actual NWS-I data. Next, base rate estimates were examined for consistency. Table 2 displays the means and standard deviations of all the taxon base rate estimates generated by the three taxometric procedures - MAXEIG, MAMBAC, and L-mode. As seen in Table 2, MAXEIG base rate estimates were quite stable $(M=0.05, S D=0.00)$. However, this consistency can be attributed to the lack of a cusp in the MAXEIG plots that prevented estimation of a base rate, instead defaulting to half of the participants in the final window as the estimate. Furthermore, base rate estimates should only be considered in combination with the plots that produce them. Thus, stability in base rate estimates alone would not be considered indicative of taxonicity. Finally, estimates were substantially lower than would be theoretically expected based on the prevalence of PTSD diagnoses in the NWS-I sample (12.3\%). 


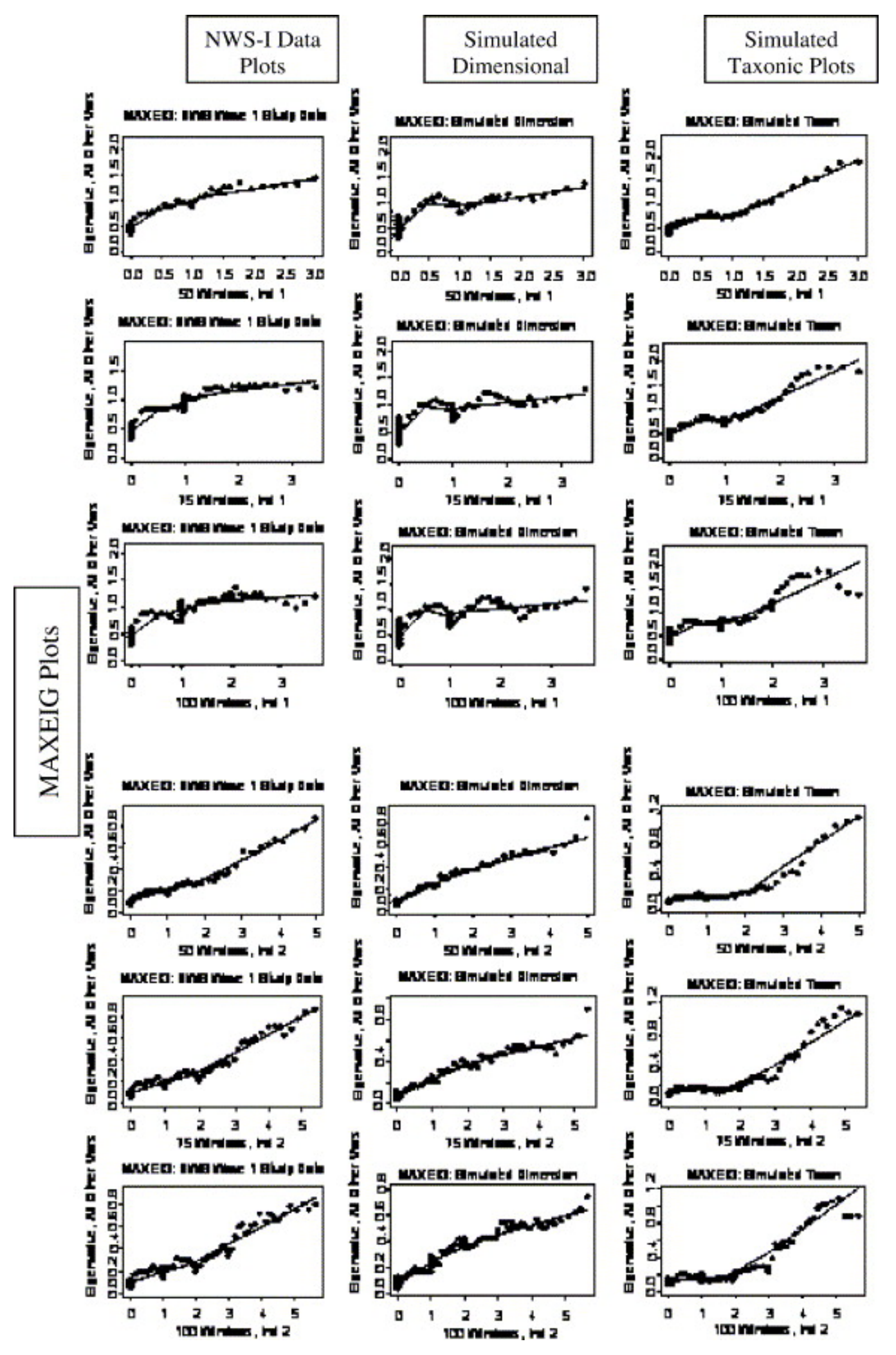

Figure 1.

MAXEIG (top), MAMBAC (middle), and L-Mode (bottom) plots for NWS-I data. 
Table 2

Means and standard deviations of the base rate estimates for NWS-I, NWS-II, and NSA data sets

\begin{tabular}{llll}
\hline Indicator & MAXEIG & MAMBAC & L-mode \\
\hline NWS-I & $.05(0.00)^{\star}$ & $.26(0.01)$ & .41 \\
NWS-II & $.05(0.00)^{*}$ & $.19(0.02)$ & .31 \\
NSA & $.05(0.00)^{*}$ & $.18(0.02)$ & .33 \\
\hline
\end{tabular}

* Lack of cusp prevented estimation of base rate, defaulting to half of the participants in the remaining window as the estimate.

One of the primary strengths and necessary components of taxometric procedures is the inherent ability to perform various consistency tests to ensure accuracy of results (Meehl, 1995). Thus, although results of the MAXEIG procedure provided support for a dimensional conceptualization of PTSD, additional taxometric procedures were conducted to determine if the results of such tests would provide consistent support for a dimensional solution. MAMBAC analyses were run next. All three of the MAMBAC plots depicted a dish shape, with a slight rise to the right, which is consistent with an underlying dimension that has been assessed using indicators with skewed distributions. The MAMBAC plots generated by the NWS-I data closely matched the simulated dimensional plots and did not evidence any peaks such as those seen in the simulated taxonic plots. The base rates produced by the MAMBAC procedure were again relatively stable $(M=0.26, S D=0.01)$, though much higher than estimates produced by MAXEIG.

L-mode was the third mathematically distinct taxometric procedure used to analyze the latent structure of PTSD in the NWS-I sample. The L-mode plot for the NWS-I data revealed a single peak, which is suggestive of dimensionality. Furthermore, the NWS-I data plot more closely matched the simulated dimensional plot than the simulated taxonic plot. The base rate estimate generated by L-mode was 0.41 , which is discrepant from estimates produced by MAXEIG and MAMBAC. Thus, L-mode results complement the findings of MAXEIG and MAMBAC in supporting a dimensional latent structure for PTSD.

\section{STUDY 2}

A potential limitation of Study 1 and the Ruscio et al. (2002) study was that the samples used in both studies were restricted to persons with a history of violent or traumatic victimization. Therefore, the purpose of Study 2 was twofold: (1) to determine whether findings relating to the latent structure of PTSD would be replicated with a follow-up sample of the NWS-I that was extended to include nonvictims as well as victims, and (2) to estimate the prevalence of PTSD in the U.S. population of adult women, should a PTSD taxon be indicated. To address these issues, MAXEIG, MAMBAC, and L-mode analyses were conducted on NWS-II data. Indicator selection and construction were identical to those implemented in Study 1. 


\section{METHOD}

\section{Participants}

Women who participated in Wave I of the NWS were recontacted 1 year later for Wave II assessment. Eighty percent of Wave I participants completed the Wave II interview $(n=3220)$; $14 \%$ could not be relocated and 6\% declined to participate. NWS-II respondents had a mean age of 44.6 years at initial assessment (SD = 17.8); $84 \%$ completed high school, $21 \%$ graduated from college, and $16 \%$ reported fewer than 12 years of education. Racial/ethnic distribution was 82.2\% Caucasian, 10.5\% African American, 4.7\% Hispanic, 1.6\% Native American, and 1.1\% Asian. Because taxometric procedures cannot handle missing data, an additional 187 women were excluded from analyses for failing to complete all PTSD items, which resulted in a final study sample of 3033.

\section{Measure}

As with Study 1, PTSD symptoms were assessed with the NWS-PTSD module (Kilpatrick et al., 1989).

\section{Procedure}

Data collection procedures were similar in focus across studies and administered by the same survey research firm (SRBI). With regard to the NWS-PTSD module, one meaningful difference occurred across studies. Whereas the PTSD module was administered only to victimized women in the NWS-I, it was administered to all participants in the NWS-II, including those who did not endorse any of the forms of victimization assessed during the interview. One of the unique features of the NWS-PTSD module is that items are not anchored to a specific victimization history, which allowed for extension of PTSD symptom assessment to nonvictims. Major advantages of this approach are that items do not require insight on the part of the respondent about symptom-event correspondence and that it facilitates straightforward assessment of symptoms with individuals who may have experienced more than one type of violent or traumatic event ( [Hanson et al., 1995] and [Resnick et al., 1993] ).

\section{Results}

The MAXEIG procedure was performed on the NWS-II data, and the ICT was employed to ensure accurate plot interpretation. Of the nine resulting MAXEIG curves, seven were relatively flat or rising to the right without evidence of a cusp, thus suggesting a dimensional solution. The remaining two curves were rated as ambiguous. Fig. 2 displays the MAXEIG, MAMBAC, and Lmode plots based on the NWS-II data and simulated taxonic and dimensional data with similar distributional characteristics. The ICT provided additional support for the absence of a latent taxon, as no peaks were evident in the plots with the highest number of windows (100 
windows). Base rate estimates were quite stable $(M=0.05, S D=0.00)$, which can again be attributed to the program defaulting to half of the participants in the final window as the estimate due to of the lack of cusping in the plots. It should also be noted that these estimates are considerably lower than would be theoretically predicted based on the prevalence of PTSD diagnoses in the general population of women (10.7\%). 

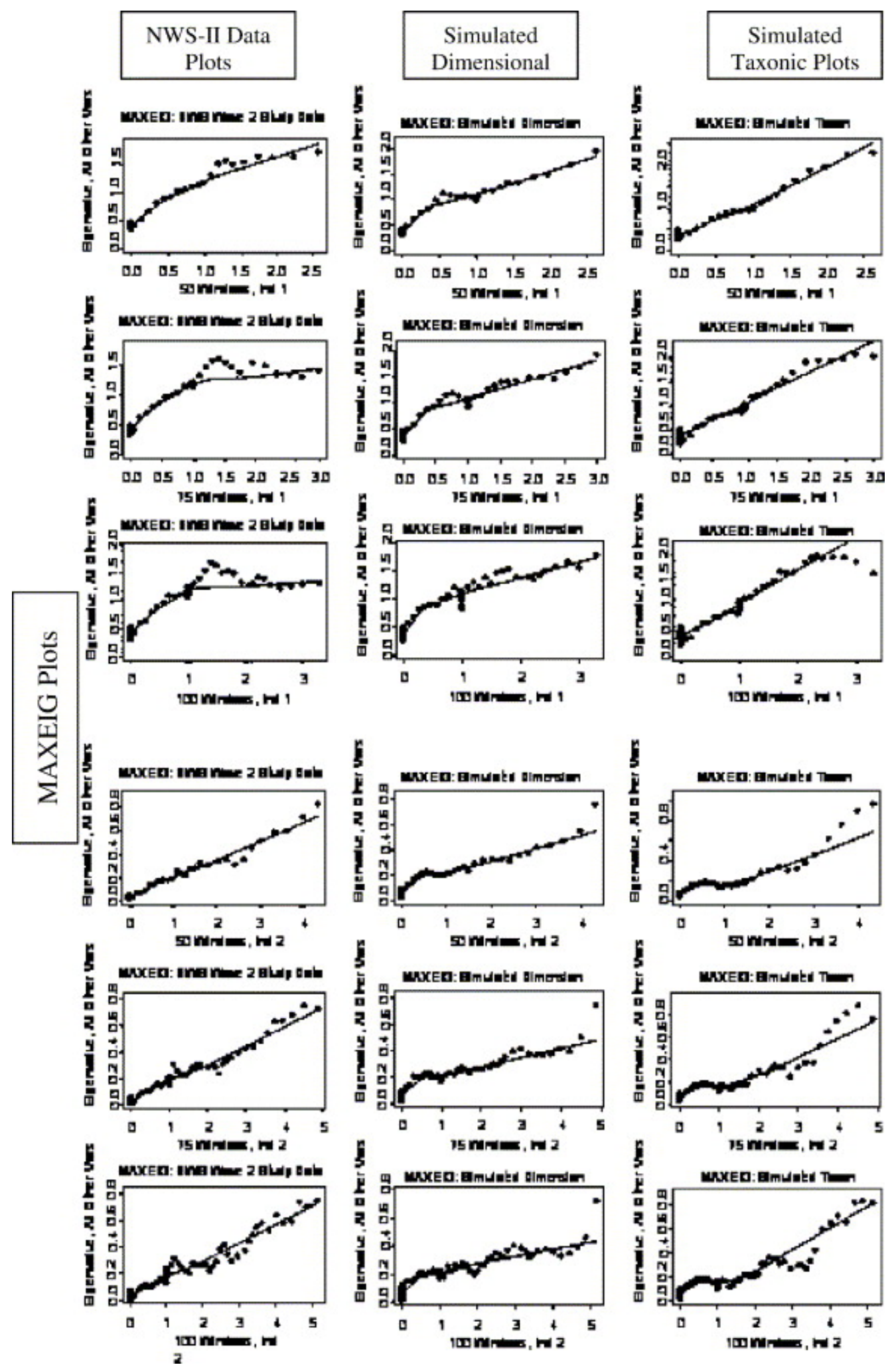

Figure 2.

MAXEIG (top), MAMBAC (middle), and L-Mode (bottom) plots for NWS-II data. 
MAMBAC analyses of the NWS-II data produced three plots, all of which evidenced the characteristic dimensional dish-shape, with slight skew to the right. NWS-II data plots were nearly identical to the simulated dimensional plots and did not display any of the peaks generated by the simulated taxonic data. Although base rates were relatively stable $(M=0.19$, $\mathrm{SD}=0.02)$, consistent base rate estimates would not be interpreted as evidence of taxonicity when the plots that produced them are dimensional.

L-mode factor analysis was the final consistency test conducted on NWS-II data. The resulting factor plot revealed a single peak and closely matched the simulated dimensional comparison. The base rate estimate was 0.31 , which is discrepant with estimates generated by MAXEIG and MAMBAC. Thus, the results of MAXEIG, MAMBAC, and L-mode analyses of the NWS-II data provide convergent evidence in support of a latent PTSD dimension.

\section{STUDY 3}

Results of Study 1, Study 2, and the Ruscio et al. (2002) analyses consistently indicated a dimensional solution for PTSD in the adult population. However, children and adolescents introduce a range of unique features to the assessment and treatment of PTSD-related reactions. Specifically, developmental factors appear to play a strong role in determining the manner with which posttraumatic stress symptoms will present ( [Amaya-Jackson and March, 1995] and [Cohen, 1998] ). Therefore, the extent to which a dimensional model of posttraumatic stress reactions would generalize to younger populations was unclear. The purpose of Study 3 was to replicate and extend Studies 1 and 2 by examining the latent structure of PTSD with a nationally representative sample of adolescents. MAXEIG, MAMBAC, and L-mode analyses were applied to data collected from the National Survey of Adolescents (NSA). Indicator selection and construction were identical to those implemented in Studies 1 and 2.

\section{METHOD}

\section{Participants}

NSA participants consisted of a national probability household sample of 3161 adolescents and a probability oversample of 862 inner-city adolescents. The sample of 3906 adolescents included 2002 boys (51.3\%) and 1904 girls (48.7\%), with a mean age of 14.5 years (SD = 1.7). The racial/ethnic breakdown was as follows: 2820 were Caucasian (72.2\%), 590 were African American (15.1\%), 311 were Hispanic (8.0\%), 139 were Native American (3.6\%), and 46 were Asian (1.2\%). Prevalences of various forms of trauma experienced were: $8.1 \%$ for sexual assault; $17.4 \%$ for physical assault; $9.4 \%$ for physically abusive punishment; and $39.4 \%$ for witnessed serious violence in the home, community, or school (see Kilpatrick, Saunders, \& Smith, 2003, for operational definitions and further details). Due to missing data on PTSD interview items, an additional 131 adolescents were excluded from analyses, resulting in a study sample of 3775 . 


\section{Measure}

The NWS-PTSD module (Kilpatrick et al., 1989) was again used to assess PTSD symptoms.

\section{Procedure}

Data collection procedures for the NSA were similar in focus to those of the NWS Waves I and $\mathrm{II}$, and sampling strategies and interviewing were administered by the same survey research firm (SRBI). A multistage, random digit dialing sampling procedure was utilized to construct the national probability sample and oversample. A detailed description of the sampling methods used to collect data for the NSA is provided elsewhere ( [Kilpatrick et al., 2000] and [Kilpatrick et al., 2003] ). As with the NWS-Il (see Study 2), the PTSD module was administered to all participants in the NSA, including those who did not endorse any of the forms of victimization assessed during the interview.

\section{Results}

MAXEIG analyses of the NSA data were conducted first. The nine plots generated by MAXEIG with the ICT were all relatively flat and consistent with those produced by simulated dimensional data. Refer to Fig. 3 for sample MAXEIG, MAMBAC, and L-mode plots based on the NSA data, as well as comparison plots generated by simulated taxonic and dimensional data with similar distributional qualities. The ICT failed to provide any evidence of a latent taxon, as no peaks emerged with increasing numbers of windows. MAXEIG base rate estimates were again consistent $(M=0.05, S D=0.00)$ due to program defaults. 


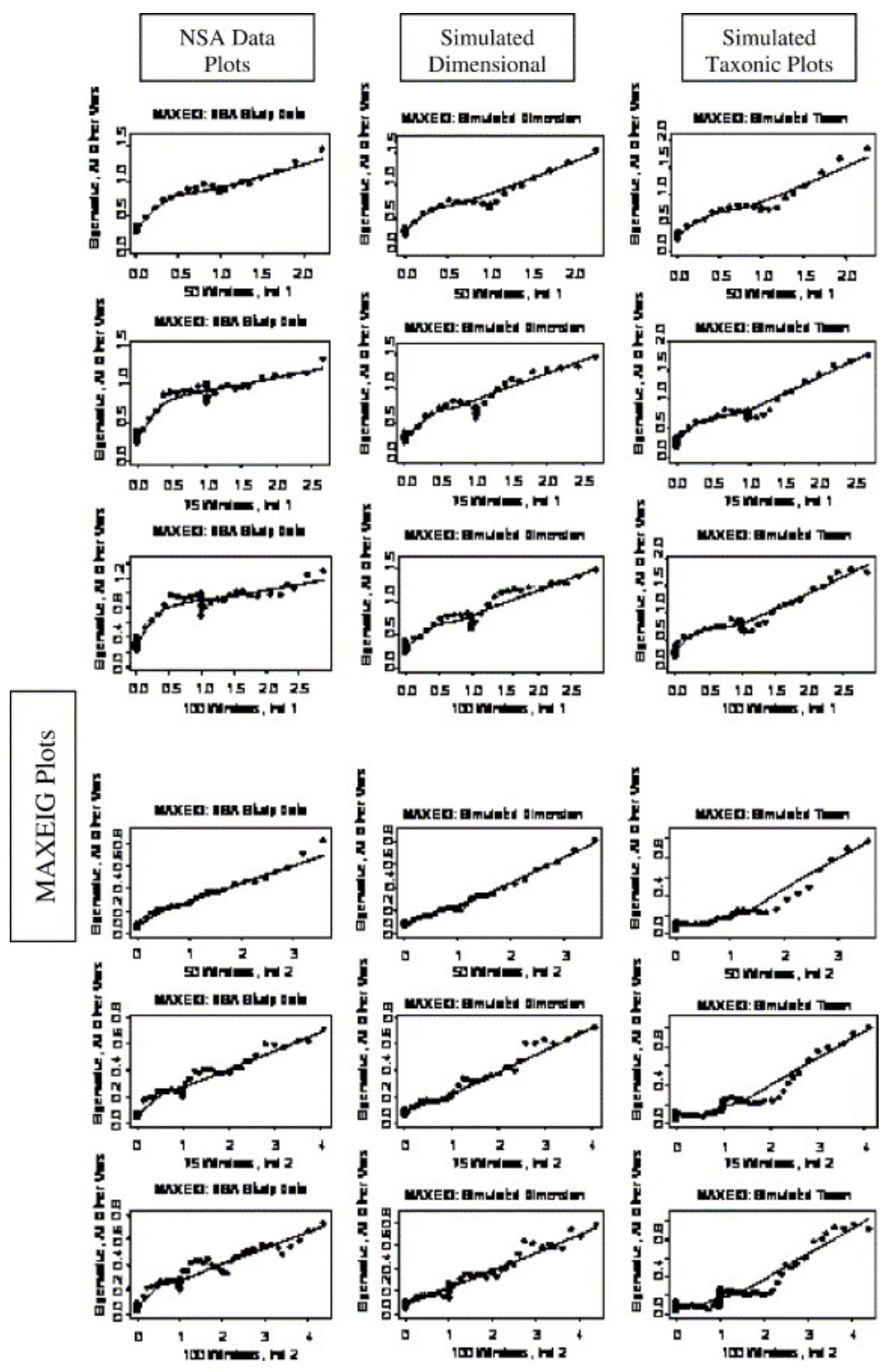

Figure 3.

MAXEIG (top), MAMBAC (middle), and L-Mode (bottom) plots for NSA data. 
MAMBAC analysis of the NSA data generated three bowl-shaped plots, each of which evidenced a slight right skew. NSA data plots were consistent with those produced by simulated dimensional data. Base rate estimates were stable $(M=0.18, S D=0.02)$, but higher than would be expected based on the prevalence of PTSD diagnoses in the adolescent sample (8.2\%).

NSA data were subjected to L-mode as the final consistency test. The probability distribution generated by L-mode evidenced a single modal peak and closely matched the simulated dimensional comparison. The base rate estimate for the NSA data was 0.33 , which again is discordant with estimates produced by MAXEIG and MAMBAC. Thus, MAXEIG, MAMBAC, and L-mode analyses of NSA data provided consistent evidence of a latent PTSD dimension.

\section{GENERAL DISCUSSION}

The purpose of this research was to examine the latent structure of PTSD using data from two large, nationally representative samples: the NWS (Waves I and II) and NSA. Previous research in this area was limited to adult, male combat veterans, thus necessitating replication of findings across more diverse samples before conclusions regarding the latent structure of PTSD could be made. Strengths associated with the current samples enabled us to replicate and extend Ruscio et al. (2002) study using multiple large representative samples comprised of adults, children, males and females, and a different measure of PTSD. In Study 1, the findings of Ruscio et al. were extended by applying three taxometric procedures-MAXEIG, MAMBAC, and L-mode-to a national sample of women with a history of one or more potentially traumatic experiences. None of these procedures produced evidence for a latent PTSD taxon. Rather, results of the three procedures converged around a dimensional solution for PTSD, as indicated by characteristically dimensional plot shapes and base rate estimates that were highly discrepant between procedures (e.g., MAXEIG versus L-Mode) and displayed wide variability around the prevalence of PTSD in the respective populations.

A potential limitation of the previous Ruscio et al. (2002) taxometric investigation was the use of a sample in which all participants had been exposed to one or more serious traumatic events (i.e., combat). It was possible that such restrictions limited the generalizability of their findings. Study 2 addressed this issue by conducting a taxometric analysis with NWS-II data, which included women both with and without a history of potentially traumatic experiences. Results of Study 2 were nearly identical to those of Study 1 and suggestive of a latent PTSD dimension. Thus, symptoms typically associated with PTSD, as currently defined by psychiatric nosology, appear to occur in various degrees among the entire adult population, regardless of trauma history, and do not demarcate a discrete psychiatric disorder.

Research has indicated that adolescents' responses to traumatic stressors can vary-sometimes considerably-from those of adults. Specifically, adolescents are more likely to present with predominately dissociative features, including depersonalization, derealization, substance abuse, and aggressive outbursts ( [Goodwin, 1988] , [Horowitz, 1996] and [Terr, 1991] ). Thus, Study 3 sought to determine whether the dimensional model for posttraumatic stress reactions that has been consistently found in adult samples would hold for a nationally representative 
sample of adolescents. Results of Study 3 were consistent with those of previous research and indicated that the latent structure of PTSD in adolescents was the same as that for adults (i.e., a dimensional structure). Thus, any differences in symptom presentations across developmental stages do not appear to represent categorical variations in the expression of posttraumatic stress reactions. Rather, a single dimension of responses to traumatic events exists for adolescents and adult men and women, with any differences reflecting differences in degree rather than differences in kind.

The findings of the present research build upon those of Ruscio et al. (2002) and improve our understanding of human responses to potentially traumatic events in several important ways. First, whereas the Ruscio et al. taxometric analysis indicated dimensional findings among clinical samples of combat veterans, the present study suggests that posttraumatic stress reactions appear to have the same dimensional structure regardless of the type of traumatic stressor. Thus, even though research has clearly indicated that certain types of extreme life events are more likely to lead to severe and persistent posttraumatic stress symptoms (e.g., rape, physical assault, combat; Kilpatrick \& Resnick, 1992), these events do not appear to affect the qualitative experience of posttraumatic stress. Rather, the same stress-response symptoms are merely experienced to varying degrees among trauma-exposed individuals.

The present findings also confirm the absence of any latent discontinuity among reactions to traumatic events between the genders. Previous research has consistently observed that women are approximately twice as likely as men to receive PTSD diagnoses even when the type of traumatic event is taken into account (Breslau, Chilcoat, Kessler, Peterson, \& Lucia, 1999). What the present research suggests is that gender does not qualitatively change posttraumatic stress symptomatology. Instead, gender is merely associated with the degree to which posttraumatic stress symptoms occur. Finally, these results suggest that human reactions to extreme stressors are relatively stable across the lifespan. Previous research has indicated that age may be associated with the frequency and intensity of posttraumatic stress symptomatology (Kessler et al., 1995), and that adolescents may even exhibit slightly different symptom patterns in response to traumatic events than adults. However, the results of the present study indicate that any differences that occur in relation to age or developmental stage do not represent a qualitatively distinct syndrome. Rather, such differences are merely variations along the same continuum of posttraumatic stress responses.

Although the present study did not look directly at subsamples defined by characteristics of the traumatic experience(s), such as severity, duration, or whether one has experienced single or multiple traumas, the current research did examine posttraumatic stress reactions in several nationally representative samples, which included a range of trauma severity and number of traumatic events. Furthermore, these data, which were consistent with U.S. census data, contained participants from diverse racial, ethnic, social, financial, and occupational backgrounds. If a posttraumatic stress taxon existed that was defined by characteristics of the traumatic stressor(s) or significantly influenced by demographic characteristics, the present taxometric analysis should have revealed its existence in these samples. However, no evidence of any taxon emerged. Thus, it can be concluded that posttraumatic stress reactions are 
continuously distributed among the general population, regardless of demographic or traumarelated characteristics.

These findings also have important implications for the assessment and study of posttraumatic stress reactions. First, a dimensional solution indicates that the use of continuous measures focused on evaluating the full range of PTSD symptom patterns and severity will maximize statistical power and minimize information loss. Measures that specifically are designed to assess PTSD diagnostic status may be strengthened in value when they allow for the computation of continuous scores to supplement existing scoring schemes. Second, a dimensional solution for posttraumatic stress reactions suggests that additive and graded etiological models of posttraumatic stress reactions are most appropriate, rather than models that suggest a specific all-or-none genetic factor or a single environmental variable (Haslam, 1997). Thus, it is likely that various trauma-related and person-specific factors (e.g., perpetrator violence, victim's interpretation, trauma history) are important in predicting the intensity and duration of an individual's posttraumatic stress reactions, and therefore should be specifically and systematically addressed in assessment and in therapy. Finally, these findings suggest that researchers should be wary of using PTSD diagnostic status as a selection or dependent variable in their designs. Rather, the PTSD literature will be best served by research designs that consider the full spectrum of PTSD symptom severity and chronicity, and use statistical procedures that analyze data across the full range of the disorder (e.g., regression analyses). At a minimum, researchers should supplement the categorical diagnosis with dimensional measures of PTSD severity.

One potential limitation of the present study warrants discussion. Specifically, the indicators that were used to evaluate the latent structure of posttraumatic stress reactions in each of the three studies were all derived from the same PTSD measure-the NWS-PTSD module. Taxometric procedures, like any other analytic procedures, are only as good as the data to which they are applied. Thus, to the extent that the NWS-PTSD module is sensitive to response style (in addition to PTSD), we run some risk of confounding our interpretation of the dimensionality of the PTSD construct with response style. However, it should be noted that the methodology and measurement of posttraumatic stress reactions utilized in the present study were quite varied from those used in the Ruscio et al. analysis, yet our findings were concordant regarding the dimensional latent structure. Future research designs that consist of varied methods of measurement across studies will improve confidence in conclusions regarding the latent structure of the variable of interest.

The present study also possessed several important strengths that enhance the confidence with which conclusions about the latent structure of PTSD can be drawn. Specifically, posttraumatic stress-related data were collected from multiple nationally representative samples of women and adolescents. Sample sizes were large and well above the minimum recommended by Meehl (1995) for detecting latent taxa. The validity of indicators was generally high and nuisance correlations were relatively low. In addition, a true multiple hurdles, multiple consistency approach to taxometrics was employed. Conclusions about the latent structure of PTSD were based on the convergent evidence provided by a variety of sources. First, indicators were subjected to three well-accepted taxometric procedures-MAXEIG, MAMBAC, and L-Mode 
factor analysis. Second, the resulting plots were compared to simulated dimensional and taxonic data with similar distributional characteristics, such as skewness and kurtosis. Third, multiple raters were employed to ensure reliable interpretation of output plots. Fourth, base rate estimates produced by each of the various procedures were analyzed for consistency both within and across procedures. Finally, an ICT was used to ensure that an extreme base rate taxon was not overlooked. Results from multiple studies and taxometric analyses converged to provide support for a model of PTSD that is nontaxonic or dimensional at the latent level.

Although debate continues among professionals regarding whether mental disorders are best represented by dimensional or categorical models, a review of the taxometric research, to date, appears to suggest that neither approach is universally accurate. Rather, certain disorders appear to be dimensional, whereas others are more accurately depicted as categorical. Based on the findings of the present study and those of Ruscio et al. (2002), it is best estimated that PTSD, as depicted by the DSM-IV, is dimensional in nature. Thus, in general, these findings appear consistent with previous research that appears to indicate that most forms of depression (e.g., [Beach and Amir, 2003] , [Franklin et al., 2002] , [Haslam and Beck, 1994] and [Ruscio and Ruscio, 2000] ) and anxiety-related disorders (e.g., Ruscio, Borkovec, \& Ruscio, 2001) have a dimensional latent structure. However, taxometric research has not yielded dimensional findings with regard to all forms of psychopathology. Specifically, several types of psychopathology, particularly those that are associated with strong biological or environmental influences, have been found to be taxonic. For example, discrete categories have been indicated with regard to schizotypy ( [Korfine and Lenzenweger, 1995] and [Lenzenweger and Korfine, 1992] ), bulimia nervosa (Gleaves et al., 2000), endogenous depression (Haslam \& Beck, 1994), and pathological dissociation ( [Waller et al., 1996] and [Waller and Ross, 1997] ). Thus, any attempts to refine psychiatric nosology should not be based on preconceived, or even "expert," notions regarding the categorical or dimensional nature of psychopathology. Rather, classification is an empirical matter that must be approached in such a way if substantial improvements are to be expected within the mental health field.

\section{REFERENCES}

Amaya-Jackson and March, 1995 L. Amaya-Jackson and J.S. March, Posttraumatic stress disorder, J.S. March, Editor, Anxiety disorders in children and adolescents, The Guilford Press, New York (1995), pp. 276-300.

American Psychiatric Association, 1994 American Psychiatric Association, Diagnostic and statistical manual of mental disorders, (4th ed.), Author, Washington, DC (1994).

Arnau et al., 2001 R.C. Arnau, R.L. Thompson and C. Cook, Do different response formats change the latent structure of responses? An empirical investigation using taxometric analysis. Educational and Psychological Measurement, 61 (2001), pp. 23-44.

Beach and Amir, 2003 S.R.H. Beach and N. Amir, Is depression taxonic, dimensional, or both?. Journal of Abnormal Psychology, 112 (2003), pp. 228-236. 
Breslau et al., 1999 N. Breslau, H.D. Chilcoat, R.C. Kessler, E.L. Peterson and V.C. Lucia, Vulnerability to assaultive violence: Further specification of the sex difference in posttraumatic stress disorder. Psychological Medicine, 29 (1999), pp. 813-821.

Cohen, 1998 J. Cohen, Summary of the practice parameters for the assessment and treatment of children and adolescents with posttraumatic stress disorder. Journal of the American Academy of Child and Adolescent Psychiatry, 37 (1998), pp. 997-1001.

Franklin et al., 2002 C.L. Franklin, D.R. Strong and R.L. Greene, A taxometric analysis of the MMPI-2 Depression Scales. Journal of Personality Assessment, 79 (2002), pp. 110-121.

Gleaves et al., 2000 D.H. Gleaves, M.R. Lowe, A.C. Snow, B.A. Green and K.P. MurphyEberez, Continuity and discontinuity models of bulimia nervosa: A taxometric investigation. Journal of Abnormal Psychology, 109 (2000), pp. 56-68.

Goodwin, 1988 J. Goodwin, Post-traumatic stress symptoms in abused children. Journal of Traumatic Stress, 1 (1988), pp. 475-488.

Grove, 1991 W.M. Grove, When is a diagnosis worth making? A statistical comparison of two prediction strategies. Psychological Reports, 68 (1991), pp. 3-17.

Hanson et al., 1995 R.F. Hanson, D.G. Kilpatrick, J.R. Freedy and B.E. Saunders, Los Angeles County after the 1992 civil disturbances: Degree of exposure and impact on mental health. Journal of Consulting and Clinical Psychology, 63 (1995), pp. 987-996.

Haslam, 1997 N. Haslam, Evidence that male sexual orientation is a matter of degree. Journal of Personality and Social Psychology, 73 (1997), pp. 862-870.

Haslam, 2003 N. Haslam, Categorical versus dimensional models of mental disorder: The taxometric evidence. Australian and New Zealand Journal of Psychiatry, 37 (2003), pp. 696704.

Haslam and Beck, 1994 N. Haslam and A.T. Beck, Subtyping major depression: A taxometric analysis. Journal of Abnormal Psychology, 103 (1994), pp. 686-692.

Horowitz, 1996 F.D. Horowitz, Developmental perspectives on child and adolescent posttraumatic stress disorder. Journal of School Psychology, 34 (1996), pp. 189-191.

Kessler et al., 1995 R.C. Kessler, A. Sonnega, E. Bromet, M. Hughs and C.B. Nelson, Posttraumatic stress disorder in the national comorbidity survey. Archives of General Psychiatry, 52 (1995), pp. 1048-1060.

Kilpatrick et al., 2000 D.G. Kilpatrick, R. Acierno, B.E. Saunders, H.S. Resnick, C.L. Best and P.P. Schnurr, Risk factors for adolescent substance abuse and dependence: Data from a national sample. Journal of Consulting and Clinical Psychology, 68 (2000), pp. 19-30.

Kilpatrick et al., 1998 D.G. Kilpatrick, H.S. Resnick, J.R. Freedy, D. Pelcovitz, P.A. Resick and S. Roth, et al. The posttraumatic stress disorder field trial: Evaluation of the PTSD construct: 
Criteria A through E, T. Widiger, A. Frances, H. Pincus, R. Ross, M. First, W. Davis, M. Kline, Editors , DSM-IV sourcebook, American Psychiatric Press, Washington, DC (1998), pp. 803844.

Kilpatrick et al., 1989 D.G. Kilpatrick, H.S. Resnick, B.E. Saunders and C.L. Best, The National Women's Study PTSD module, National Crime Victims Research and Treatment Center, Medical University of South Carolina, Charleston, SC (1989).

Kilpatrick et al., 2003 D.G. Kilpatrick, K.J. Ruggiero, R. Acierno, B.E. Saunders, H.S. Resnick and C.L. Best, Violence and risk of PTSD, major depression, substance abuse/dependence, and comorbidity: Results from the National Survey of Adolescents. Journal of Consulting and Clinical Psychology, 71 (2003), pp. 692-700.

Kilpatrick et al., 2003 D.G. Kilpatrick, B.E. Saunders and D.W. Smith, Youth victimization: Prevalence and implications. Research in Brief, National Institute of Justice, Washington, DC (2003).

Korfine and Lenzenweger, 1995 L. Korfine and M.F. Lenzenweger, The taxonicity of schizotypy: A replication. Journal of Abnormal Psychology, 104 (1995), pp. 26-31.

Lenzenweger and Korfine, 1992 M.F. Lenzenweger and L. Korfine, Confirming the latent structure and base rate of schizotypy: A taxometric analysis. Journal of Abnormal Psychology, 101 (1992), pp. 567-571.

Marshall et al., 2001 R.D. Marshall, M. Olfson, F. Hellman, C. Blanco, M. Guardino and E.L. Struening, Comorbidity, impairment, and suicidality in subthreshold PTSD. American Journal of Psychiatry, 158 (2001), pp. 1467-1473.

Meehl, 1973 P.E. Meehl, MAXCOV-HITMAX: A taxonomic search method for loose genetic syndromes, P.E. Meehl, Editor, Psychodiagnosis: Selected papers, University of Minnesota Press, Minneapolis (1973), pp. 200-224.

Meehl, 1995 P.E. Meehl, Bootstraps taxometrics: Solving the classification problem in psychopathology. American Psychologist, 50 (1995), pp. 266-275.

Meehl and Golden, 1982 P.E. Meehl and R.R. Golden, Taxometric methods, J.N. Butcher, P.C. Kendall, Editors, The handbook of research methods in clinical psychology, Wiley, New York (1982), pp. 127-181.

Meehl and Yonce, 1994 P.E. Meehl and L.J. Yonce, Taxometric analysis I: Detecting taxonicity with two quantitative indicators using means above and below a sliding cut (MAMBAC procedure). Psychological Reports, 74 (1994), pp. 1059-1274.

Meehl and Yonce, 1996 P.E. Meehl and L.J. Yonce, Taxometric analysis II: Detecting taxonicity using covariance of two quantitative indicators in successive intervals of a third indicator (MAXCOV procedure). Psychological Reports, 78 (1996), pp. 1091-1227. 
Norris, 1992 F. Norris, Epidemiology of trauma: Frequency and impact of different potentially traumatic events on different demographic groups. Journal of Consulting and Clinical Psychology, 60 (1992), pp. 409-418.

Resnick et al., 1993 H.S. Resnick, D.G. Kilpatrick, B.S. Dansky, B.E. Saunders and C.L. Best, Prevalence of civilian trauma and posttraumatic stress disorder in a representative national sample of women. Journal of Consulting and Clinical Psychology, 61 (1993), pp. 984-991.

Rothbaum et al., 1992 B.O. Rothbaum, E.B. Foa, D.S. Riggs, T. Murdock and W. Walsh, A prospective examination of post-traumatic stress disorder in rape victims. Journal of Traumatic Stress, 5 (1992), pp. 455-475.

Ruggiero et al., 2003 K.J. Ruggiero, H.S. Resnick, S. Galea, D.G. Kilpatrick, A.A. Rheingold and J. Ahern, et al. National Women's Study PTSD Module and PTSD Checklist: A comparison of instruments Poster presented at the 37th annual convention of the Association for Advancement of Behavior Therapy, Boston (2003, November).

Ruscio et al., 2001 A.M. Ruscio, T.D. Borkovec and J. Ruscio, A taxometric investigation of the latent structure of worry. Journal of Abnormal Psychology, 110 (2001), pp. 413-422.

Ruscio and Ruscio, 2002 A.M. Ruscio and J. Ruscio, The latent structure of analogue depression: Should the Beck Depression Inventory be used to classify groups?. Psychological Assessment, 14 (2002), pp. 135-145.

Ruscio et al., 2002 A.M. Ruscio, J. Ruscio and T.M. Keane, The latent structure of posttraumatic stress disorder: A taxometric investigation of reactions to extreme stress. Journal of Abnormal Psychology, 111 (2002), pp. 290-301.

Ruscio, 2003 Ruscio, J. (2003). Documentation of program code to perform taxometric analyses and simulate comparison data in the $\mathrm{R}$ and $\mathrm{S}+$ languages. Retrieved July 1,2003 , from http://www.etown.edu/psychology/faculty/ruscio.htm.

Ruscio and Ruscio, 2000 J. Ruscio and A.M. Ruscio, Informing the continuity controversy: A taxometric analysis. Journal of Abnormal Psychology, 109 (2000), pp. 473-487.

Spitzer et al., 1992 R.L. Spitzer, J.B. Williams, M. Gibbon and M.B. First, The structured clinical interview for DSM-III-R (SCID): I. History, rationale, and description. Archives of General Psychiatry, 49 (1992), pp. 624-629.

Stein et al., 1997 M.B. Stein, J.R. Walker, A.L. Hazen and D.R. Forde, Full and partial posttraumatic stress disorder: Findings from a community survey. American Journal of Psychiatry, 154 (1997), pp. 1114-1119.

Terr, 1991 L.C. Terr, Childhood traumas: an outline and overview. American Journal of Psychiatry, 50 (1991), pp. 10-20. 
Veronen and Kilpatrick, 1983 L.J. Veronen and D.G. Kilpatrick, Stress management for rape victims, D. Meichenbaum, M.E. Jaremko, Editors , Stress reduction and prevention, Plenum, New York (1983), pp. 341-374.

Waller and Meehl, 1998 N.G. Waller and P.E. Meehl, Multivariate taxometric procedures: Distinguishing types from continua, Sage, Newberry Park, CA (1998).

Waller et al., 1996 N.G. Waller, F.W. Putnam and E.B. Carlson, Types of dissociation and dissociative types: A taxometric analysis of dissociative experiences. Psychological Methods, 1 (1996), pp. 300-321.

Waller and Ross, 1997 N.G. Waller and C.A. Ross, The prevalence and biometric structure of pathological dissociation in the general population: Taxometric and behavior genetic findings. Journal of Abnormal Psychology, 106 (1997), pp. 499-510. 